\title{
Gasto Energético Corporal: Conceitos, Formas de Avaliação e sua Relação com a Obesidade
}

\section{revisão}

\author{
Camila Maria de Melo \\ JULIO TIRAPEGUI \\ SANDRa MARIa Lima Ribeiro \\ Faculdade de Ciências \\ Farmacêuticas (FCF) da \\ Universidade de São Paulo (USP), \\ $\mathrm{SP}$, Brasil (CMM, JT); Escola de \\ Artes, Ciências e Humanidades \\ $(\mathrm{EACH})$ da USP (SMLR), SP, Brasil.
}

Recebido em 12/07/2007 Aceito em 21/01/2008

\section{RESUMO}

A obesidade, apesar de intensamente estudada nos últimos anos, ainda apresenta muitas controvérsias, entre elas a sua relação com o gasto energético (GE). O presente estudo representa uma revisão da literatura procurando abordar: as técnicas existentes para avaliação do GE, com suas vantagens e limitações e as relações existentes entre GE e a obesidade. O GE pode ser avaliado por métodos calorimétricos, espectrométricos, ou mesmo por questionários. De acordo com os objetivos e as possibilidades da pesquisa, todas as técnicas apresentam pontos positivos e limitações. Os indivíduos obesos têm sido apontados como "econômicos", do ponto de vista metabólico, ou seja, gastariam menos energia do que os não obesos. Essa economia tem sido investigada a partir de cada componente do GE diário (GED) (GE de repouso, efeito termogênico à alimentação ou GE da atividade física) e os resultados são bastante controversos. Paralelamente às análises do GE em humanos, estudos moleculares têm auxiliado no esclarecimento desse tema tão complexo. Diferentes hormônios, especialmente os secretados pelo adipócito, bem como proteínas específicas em vários tipos de células, têm auxiliado na compreensão do GE corporal. Muitos estudos ainda são necessários, e é importante que se padronizem as técnicas, de maneira que se permita maior reprodutibilidade dos resultados. (Arq Bras Endocrinol Metab 2008; 52/3:452-464)

Descritores: Gasto energético; Obesidade; Avaliação

\section{ABSTRACT}

Human Energetic Expenditure: Concepts, Assessment Methods and Relationship to Obesity.

Despite many studies in the last years, obesity still needs more investigation, including its relation with energetic expenditure (EE). This manuscript consisted of a review considering: the existing techniques to measure $E E$, showing their advantages and limitations and modifications in EE due to obesity. EE can be evaluated by calorimetric methods, spectroscopic methods, or by questionnaires. According to the research's objectives and possibilities, all techniques present advantages and limitations. Obese individuals have been recognized as "economics" in metabolic point of view, which means that they are able to expend little energy, compared to non-obese individuals. This economy has been investigated in many aspects. They have been analyzed every component of daily energetic expenditure (resting $E E$, thermogenic response to feeding and EE by physical activity), and the results are controversial, mainly because of the lack on standardization of procedures and techniques. Together with evaluation of $\mathrm{EE}$ in humans, molecular studies have contributed to clarify many aspects. They have been identified hormones and cellular proteins located in different kinds of cells. Studies are still necessary, and it is imperative to standardize the procedures and techniques to allow the reproducibility of the results. (Arq Bras Endocrinol Metab 2008; 52/3:452-464)

Keywords: Energetic expenditure; Obesity; Evaluation 


\section{INTRODUÇÃO}

S CAUSAS E AS CONSEQÜÊNCIAS da obesidade têm sido extensamente estudadas nos últimos anos, principalmente por sua relação com transtornos psicológicos e comorbidades (1-4). É importante destacar que o Brasil, da mesma forma que vários outros países, tem apresentado, nos últimos anos, aumento da prevalência da obesidade e redução no déficit de peso (5).

A obesidade é causada pela combinação entre predisposição genética e estilo de vida. A inatividade física e a alimentação inadequada resultam balanço energético (BE) positivo, o que significa em última instância aumento do peso corporal $(6,7)$. A complexidade da regulação do peso corporal representa um dos maiores desafios para o entendimento da etiologia, tratamento e prevenção da obesidade (8). Neste contexto, muitos conhecimentos são necessários, desde a compreensão dos métodos apropriados para avaliação do $\mathrm{BE}$ nos indivíduos, até as técnicas bioquímicas e moleculares que possam esclarecer os mecanismos específicos.

Considerando os aspectos abordados anteriormente, o presente estudo consistiu uma revisão da literatura com os objetivos de identificar as técnicas existentes para avaliação do gasto energético (GE), com suas vantagens e limitações, e identificar as alterações no GE associadas à obesidade e suas possíveis explicações, apresentando as diferentes técnicas utilizadas nos estudos.

\section{MATERIAIS E MÉTODOS}

O trabalho consistiu de uma revisão da literatura, com consulta às seguintes bases de dados: Medline, Lilacs $\mathrm{e}$ PubMed. A busca foi realizada com os seguintes unitermos: gasto energético, obesidade, peso corporal, exercício físico, efeito térmico da alimentação, termogênese. O período de consulta foi entre 1995 a 2005. Quando foram identificadas nos artigos citações relevantes anteriores ao período consultado, estas também foram obtidas e incluídas. Para esclarecimento de alguns conceitos básicos, foram consultados alguns livros didáticos.

\section{DESENVOLVIMENTO}

O total de energia necessário para os seres vivos, ou o gasto energético diário (GED) compreende o dispêndio energético basal (GEB), necessário para a realização das funções vitais do organismo; o gasto energético da atividade física (GEAF), que engloba as atividades físicas do cotidiano e o exercício físico; e o efeito térmico dos alimentos (ETA), relacionado com a digestão, a absorção e o metabolismo dos alimentos. Em indivíduos saudáveis, o GEB corresponde aproximadamente a $60 \%$ a $70 \%$ do gasto diário, o ETA entre $5 \%$ e $15 \%$ e o GEAF de $15 \%$ a $30 \%$, sendo este último o componente que mais varia entre os indivíduos (9-13).

\section{MÉTODOS PARA AVALIAÇÃO DO GASTO ENERGÉTICO}

Dada a importância de se investigar os aspectos do GE nos estudos sobre a obesidade, os principais métodos $\mathrm{e}$ as técnicas serão descritos a seguir.

\section{Calorimetria direta}

Essa técnica deriva dos estudos inicias de Lavoisier e Laplace, seguidos por Atwater e Rosa e Atwater e Benedict, que a desenvolveram e aperfeiçoaram para avaliação do GE em humanos (14). A calorimetria direta requer uma câmara altamente sofisticada, que permite a medida do calor sensível liberado pelo organismo, além do vapor de água liberado pela respiração e pela pele (15). Para a avaliação do GED, o avaliado deve permanecer na câmara por período igual ou superior a 24 horas (16).

\section{Calorimetria indireta}

A calorimetria indireta mensura o GE por meio da análise do oxigênio consumido $\left(\mathrm{VO}_{2}\right)$, do gás carbônico produzido $\left(\mathrm{VCO}_{2}\right)$ e, ainda, do quociente respiratório $(\mathrm{QR}$ $\left.=\mathrm{VO}_{2} / \mathrm{VCO}_{2}\right)$, apontando assim a quantidade de energia necessária para a realização dos processos metabólicos. É considerada uma técnica de custo razoável, não invasiva e com grande reprodutibilidade (11). Pode ser desenvolvida de duas formas diferentes: por circuito fechado e por circuito aberto. Na primeira, o indivíduo é conectado a uma máscara por meio da qual ele respira o ar com composição conhecida, vindo de um cilindro, e volta a respirar somente o ar do espirômetro. $\mathrm{O}$ consumo de oxigênio pode ser determinado a partir da quantidade removida do sistema. Essa técnica não permite ao avaliado muita mobilidade e, por isso, é utilizada prioritariamente para situações de repouso (15). Outra possibilidade para essa análise é a utilização da chamada câmara respiratória ou calorímetro de sala. Nela, o indivíduo reside por período de aproximadamente 24 horas, 
similarmente à calorimetria direta, podendo realizar quase todas as suas atividades diárias. É medida a troca gasosa sem a medida da produção de calor (17). Um exemplo dessa câmara encontra-se na Lausanne University, Suiça (18). O grande inconveniente dessa alternativa é o alto custo do equipamento.

$\mathrm{Na}$ calorimetria indireta de circuito aberto, o avaliado respira por uma válvula de duas vias, por uma das quais é inspirado o ar ambiente, e por outra o ar expirado é coletado e analisado. Essa análise pode ser feita em tempo real (instrumentação computadorizada) ou pode ser armazenada para análise posterior (espirometria portátil ou técnica de bolsa) (19). Essa análise é feita em intervalos determinados e depois os valores são extrapolados para as 24 horas do dia, a partir de relações e fórmulas específicas.

A determinação do GEB por calorimetria indireta necessita que a medida seja feita durante o período de sono do avaliado. Pela dificuldade de se medir o indivíduo nessa situação, grande parte dos estudos na literatura utilizam a medida do gasto energético de repouso (GER), que é feita geralmente pela manhã com o indivíduo deitado, porém acordado (14). Ainda, por meio da calorimetria indireta também se pode avaliar a capacidade física dos indivíduos. Os chamados testes ergoespirométricos geralmente são feitos no intuito de se avaliar o consumo máximo de oxigênio $\left(\mathrm{VO}_{2}\right.$ máximo), e a partir deste pode-se predizer o grau de condicionamento físico dos indivíduos e prescrever exercícios físi$\cos (19,20)$.

Por sua praticidade, a calorimetria indireta de circuito aberto é o método calorimétrico mais utilizado, tanto em pesquisas quanto na prática clínica. Entretanto, é difícil, a partir dessa análise, determinar o GE de todas as atividades realizadas durante o dia $(21,22)$. Uma possibilidade para o detalhamento dessas atividades pode ser a aplicação paralela de questionários que descrevam as atividades diárias.

\section{Questionários para avaliação do gasto energético diário}

Para que se possa ter uma dimensão ampla do GE pela atividade física diária, é necessário que se relatem todos os tipos de atividade realizados durante o dia, com o maior grau de detalhamento possível. Embora esse método seja cercado de subjetividade, em grande parte das vezes acaba sendo um método complementar de grande importância.
Existem questionários que podem ser definidos como indiretos, nos quais, com base no relato do tempo e do grau de percepção do esforço de categorias de atividade, se considera o nível de atividade do indivíduo. Um exemplo desse tipo de questionário é o IPAQ (International Physical Activity Questionary), desenvolvido nos Estados Unidos (23) e validado para a população brasileira (24). Outros tipos de questionário, a partir dos relatos detalhados de diários de cada atividade física realizada e da intensidade percebida, também permitem o cálculo da energia gasta diariamente $(25)$.

\section{Avaliação do GE com base no consumo alimentar}

Durante muitos anos, a determinação do GE dos indivíduos foi feita com base naquilo que era ingerido pela dieta. Considerava-se que, se o indivíduo estivesse com o peso e a composição corporal adequados, realizando todas as atividades diárias de maneira satisfatória, a medida de sua ingestão habitual forneceria uma noção de seu gasto de energia (26). Entretanto, a avaliação da ingestão alimentar está sujeita a grande gama de erros, tanto nos relatos quanto nos cálculos do valor energético do alimento. Além disso, a regulação da energia pelo homem não pode ser considerada um processo perfeito, sendo sujeita a grandes flutuações $(27,28)$.

\section{Equações preditivas do GE}

Em 1919, Harris e Benedict deduziram os primeiros dados de GEB a partir de equações de regressão obtidas por estudos de calorimetria. A partir da década de 1980, retomaram-se os estudos de GE com indivíduos saudáveis, quando a Organização Mundial da Saúde publicou novas equações para predição do GEB, além de estabelecer múltiplos para predição do GED $(14,29,30)$.

As equações de predição são métodos rápidos e fáceis, além de terem baixo custo. Por outro lado, requerem a estimativa do GE das atividades físicas realizadas durante o dia, o que implica a necessidade dos relatos da atividade física diária. Além disso, muitas dessas equações foram elaboradas por um grupo de pessoas com faixa etária estreita e com indivíduos brancos, $\mathrm{o}$ que geralmente não representa especificamente todos os segmentos da população (31). O comitê de especialistas responsáveis pelas atuais Dietary Reference Intakes (32), procurando aprimorar as predições de GE, tem 
proposto novos modelos de equações, a partir da metodologia da água duplamente marcada.

\section{Sensores de movimento}

Os sensores de movimento são fundamentados na acelerometria. Consistem de dispositivos que são fixados em partes específicas do corpo, capazes de medir os movimentos em até três eixos corporais: ântero-posterior, lateral e vertical. Este método de determinação possui as vantagens de poder avaliar o indivíduo em sua vida cotidiana, além do baixo custo. Por outro lado, é necessária uma escolha apropriada do local de fixação do dispositivo, pois há o risco de o dispositivo se soltar do corpo (33-36).

\section{Água duplamente marcada}

Atualmente, o método considerado como padrão-ouro para determinação do GE é a água duplamente marcada. Esta técnica, inicialmente aplicada somente em pequenos animais, permite medir o GE de indivíduos fora de confinamento, sem necessidade de nenhuma modificação no cotidiano e sem necessidade de fixação de dispositivos ao corpo $(37,38)$. O indivíduo ingere uma dose de água marcada com isótopos não-radioativos de oxigênio e hidrogênio $\left({ }^{18} \mathrm{O}\right.$ e $\left.{ }^{2} \mathrm{H}\right)$. Pelo princípio do método, o isótopo de oxigênio é eliminado do corpo incorporado nas moléculas de dióxido de carbono e água. Por sua vez, o isótopo de hidrogênio é eliminado somente como água. Assim, a diferença na eliminação entre esses dois isótopos ingeridos simultaneamente pode predizer a medida da produção de gás carbônico e, assim, indiretamente, o $\operatorname{GE}(17,22,38,39)$. A água duplamente marcada pode medir o GE total dos indivíduos por períodos entre uma a duas semanas.

Este método é capaz de medir o GED, porém não mede o nível de atividade física dos indivíduos. Sendo assim, a aplicação de diários de atividade física complementa e esclarece as informações obtidas, da mesma forma que para a calorimetria indireta (40). A grande limitação para o método reside em seus custos, tanto relativos ao equipamento necessário (espectrômetro de massa) quanto aos isótopos (31). Por outro lado, cabe destacar que este método tem fornecido resultados com precisão e objetividade na investigação das questões relativas ao GE e à obesidade (41).

Existem ainda outras técnicas propostas para avaliação do GE em humanos, menos utilizadas, e que por isso não serão abordadas nesta revisão $(40,42)$.

\section{A importância das medidas hormonais e das técnicas moleculares}

Com os avanços da biologia molecular, tem-se buscado a compreensão não somente do total de energia gasto pelo indivíduo, mas também das origens moleculares desse gasto. Nesse sentido, vale destacar a identificação de várias moléculas sintetizadas pelos adipócitos, além de proteínas de membrana na mitocôndria e no núcleo de várias células, que muito têm colaborado com essa compreensão (43-46).

\section{RELAÇÃO ENTRE GE E OBESIDADE}

Vários estudos mostram que animais, quando sujeitos a períodos de restrição alimentar, aumentam sua capacidade de armazenar nutrientes, melhorando sua eficiência metabólica (47-50). Em humanos, a existência desse possível sistema de auto-regulação poderia ser explicada pelo período em que os homens necessitavam caçar seu próprio alimento, estando, portanto, relacionada à sobrevivência $(13,51)$. Por outro lado, a manutenção de um BE positivo cronicamente, resultando aumento no peso corporal, parece não promover aumentos proporcionais no GE. Isso sugere que nosso organismo é programado para proteger-se mais intensamente contra a perda do que contra o ganho de peso corporal (8). Não se compreende ao certo por que a maioria dos obesos não consegue perder peso e, quando o perde, acabam por retomar seu peso anterior. Um dos fatores preocupantes nesse contexto é que a diminuição do GE nos períodos de restrição alimentar possivelmente se mantém mesmo após o retorno da ingestão energética habitual (45).

O curso das investigações sobre GE diminuído na obesidade aponta para duas possibilidades: obesos naturalmente têm seu GE diminuído e indivíduos obesos que foram submetidos, em diferentes períodos da vida, a restrições alimentares, ajustam seu GE e se tornam mais econômicos. Major e cols. (52) chamam este fenômeno de termogênese adaptativa.

Considerando a primeira possibilidade, por muito tempo se acreditou que indivíduos obesos tivessem um GE menor do que não-obesos (41). Alguns estudos iniciais, como o de Keen e cols. (53) e Braitman e cols. (54), analisaram o consumo energético por meio de diários alimentares e mostraram que este era menor em indivíduos com maior adiposidade, relacionando, portanto, a obesidade à redução no GE (41). Shah e cols. (55), 
utilizando calorimetria indireta, compararam indivíduos magros e pós-obesos, e calcularam a termogênese facultativa $(\mathrm{TF})$, definida como TF $=$ GED $-($ GEAF + GER $)$. Essa termogênese foi menor nos indivíduos pós-obesos, indicando que estes teriam maior capacidade de armazenar energia. A termogênese foi ainda menor nos indivíduos que relataram a presença de obesidade familiar, indicando também uma característica genética relacionada a esses aspectos.

De outra parte, estudos mais recentes afirmam que indivíduos obesos, ao contrário do que afirmaram as pesquisas anteriores, têm um GE total maior que indivíduos magros $(56,57)$. As evidências indicam que grande parte dos estudos que discutiam o GE na obesidade comparava o consumo alimentar diário com a estimativa do GED. Hoje, sabe-se que a subnotificação do consumo alimentar é freqüente em obesos, com prevalência que varia entre $20 \%$ e $50 \%(28,58$ 60). Assim, a falta de veracidade nos inquéritos alimentares pode ter sido a causa pela qual grande parte dos estudos concluiu que obesos tinham o GE diminuído. A tendência atual dos estudos tem sido concordar muito mais com a hipótese da termogênese adaptativa do que com a possibilidade de obesos terem naturalmente seu GE reduzido. Os estudos a seguir investigam essa possibilidade.

Leibel e cols. (61) estudaram as mudanças no GE em 18 indivíduos obesos e 23 indivíduos que nunca foram obesos. Estes indivíduos foram avaliados nas seguintes situações: a) peso inicial; b) após ganho de 10\% de peso corporal; após perda de $10 \%$ a $20 \%$ do peso inicial. $\mathrm{Na}$ fase de perda de peso, foi encontrada redução no GER de 52,7 a 69,8 kcal/kg de massa magra no grupo de indivíduos obesos. Esse achado pode representar uma mudança compensatória do organismo, tentando retornar ao seu peso inicial. Doucet e cols. (62) avaliaram, por calorimetria indireta e por equações preditivas, a resposta do GER em homens e mulheres obesos após um programa de restrição energética. Os resultados mostraram maior redução no GER pela calorimetria do que pelas fórmulas preditivas, apontando também essa possível adaptação. No estudo Biosphere 2 foi avaliado o GE por calorimetria indireta em indivíduos submetidos a dois anos de restrição calórica. Os resultados mostraram que a taxa metabólica de repouso dos participantes, que perderam em média $15 \%$ do peso corporal, foi menor do que o grupo controle, mesmo quando corrigidos por idade, sexo, gordura e massa magra (63). O Minnesota
Experiment, submeteu 32 voluntários homens com peso corporal normal ao seguinte protocolo experimental 24 semanas de semijejum, seguidos de 12 semanas de realimentação restritiva e mais oito semanas com alimentação ad libitum. $\mathrm{O}$ resultado nas últimas semanas foi direcionado para comportamentos hiperfágicos e elevação das reservas de gordura corporal. Durante os períodos de restrição alimentar, a diminuição no GEB foi proporcional à perda de gordura corporal, e as alterações persistiram, mesmo quando o peso corporal retornou ao estado inicial (64).

Por outro lado, vários estudos se contrapõem à proposta da termogênese adaptativa. Weinsier e cols. (65) compararam o GE entre mulheres brancas e negras, antes e após a perda de peso, por calorimetria de sala e por água duplamente marcada. Os autores observaram que a diminuição no GE, após a perda de peso, deveu-se exclusivamente à perda de massa magra, o que nega a hipótese de adaptação. Os mesmos autores, em outro estudo (57), também não encontraram diferenças no GER e no GED em mulheres com peso normal comparadas com mulheres ex-obesas com grande predisposição à obesidade. Wyatt e cols. (51) submeteram um grupo de indivíduos à perda de peso, e não encontraram nenhuma indicação de aumento na eficiência energética. Todavia, por causa da amostra consistir apenas de indivíduos que obtiveram sucesso na perda e manutenção do peso, pode-se deduzir que a seleção da amostra pode ter sido tendenciosa. Ainda, Doucet e cols. $(62,66)$, estudando homens e mulheres submetidos à perda de peso durante 15 semanas, mostraram que o GER somente foi alterado significativamente para os homens e não para mulheres, visto que ambos os grupos perderam massa magra e gordura corporal. Outros estudos relacionando perda de peso e GE estão descritos na Tabela 1 .

O que se pode observar, com base nas diferenças desses estudos, é que existe grande variabilidade individual a esses ajustes metabólicos, o que torna bastante difícil a explicação para eles (72).

Tentando solucionar as dificuldades de se interpretar o GE por técnicas clássicas, vários autores têm buscado explicações moleculares para a existência da termogênese adaptativa ou, ainda, para avaliar a possibilidade de indivíduos obesos diminuírem naturalmente seu gasto de energia. Dulloo e Jacquet (73) discutem, entre outros fatores, o controle exercido pelo tecido adiposo, que pode sinalizar outras regiões do organismo. Metabolicamente, o excesso de gordura corpórea pode gerar um estado pró-inflamatório, contribuindo 
Tabela 1. Estudos relacionando perda de peso e GE.

\begin{tabular}{|c|c|c|}
\hline Autor & Métódos* & Resultados* \\
\hline $\begin{array}{l}\text { Kempen e cols., } \\
1995(67)\end{array}$ & $\begin{array}{l}20 \text { mulheres obesas submetidas a } 8 \text { semanas } \\
\text { de } R C \\
\text { ou } R C \text { + exercício físico (aeróbio + contra- } \\
\text { resistência). Avaliados no início e no final do } \\
\text { programa: GET, } \\
\text { GER e GEAF. }\end{array}$ & $\begin{array}{l}\text { Diminuíram PC, MM e MG }(p<0,0001) \text {, no GER } \\
\text { e no GET nos dois tratamentos. Correlação } \\
\text { significativa e positiva entre o GET e o GER } \\
\text { com a massa magra }(p<0,05) \text {. }\end{array}$ \\
\hline Leibel e cols., 1995 (61) & $\begin{array}{l}18 \text { indivíduos obesos e } 23 \text { magros. Avaliações: } \\
\text { GET, GER, GEAT e ETA, no peso inicial, após RC } \\
\text { com } 10 \% \text { do peso corporal, e após ganho de } \\
10 \% \text { do peso corporal. }\end{array}$ & $\begin{array}{l}\text { Redução no GET, no GER e no GEAF com a } \\
\text { perda de peso. Aumento no GET, no GER, no } \\
\text { GEAF e no ETA com o aumento do peso } \\
\text { corporal. }\end{array}$ \\
\hline Foster e cols., 1999 (68) & $\begin{array}{l}198 \text { mulheres obesas, brancas e negras, } \\
\text { sobrepesadas; comparação de exercício e } \\
\text { exercício + RC. }\end{array}$ & $\begin{array}{l}\text { Diminuição do GER ( } 9,9 \% \text { negras e } 6,3 \% \\
\text { brancas) nos dois grupos com a perda de } \\
\text { peso. }\end{array}$ \\
\hline $\begin{array}{l}\text { Doucet e cols., } \\
2000(66)\end{array}$ & $\begin{array}{l}40 \text { indivíduos, dois grupos: tratamento } \\
\text { medicamentoso e RC por } 15 \text { semanas. } \\
\text { Avaliações: composição corporal, GER e } \\
\text { alguns hormônios. }\end{array}$ & $\begin{array}{l}\text { Redução no PC, na MM e na MG para } \\
\text { homens e mulheres. Redução de GER de } 13 \% \\
\text { nos homens }(p<0,01) \text { e na oxidação lipídica } \\
\text { de } 11 \%(p=0,08) \text {. }\end{array}$ \\
\hline $\begin{array}{l}\text { Doucet e cols., } \\
2001(62)\end{array}$ & $\begin{array}{l}15 \text { homens e } 20 \text { mulheres; dois grupos: } \\
\text { tratamento medicamentoso e RC por } 15 \\
\text { semanas. Avaliados: GER e composição } \\
\text { corporal durante e no final. }\end{array}$ & $\begin{array}{l}\text { Nas semanas } 2 \text { e } 8 \text { houve } \\
\text { redução além do predito no GER nos dois } \\
\text { grupos. Essa redução se manteve no final } \\
\text { para os homens. }\end{array}$ \\
\hline $\begin{array}{l}\text { Weinsier e cols., } \\
2003(57)\end{array}$ & $\begin{array}{l}98 \text { mulheres com peso normal, sendo } 49 \\
\text { submetidas } \\
\text { à restrição calórica de } 800 \mathrm{kcal} / \text { dia Foi } \\
\text { avaliado: composição corporal (DEXA), GE } \\
\text { (calorimetria } \\
\text { direta) e QR. }\end{array}$ & $\begin{array}{l}\text { Após um ano, não houve diferença no GER, } \\
\text { no GEB e no GET entre os grupos. }\end{array}$ \\
\hline $\begin{array}{l}\text { Doucet e cols., } \\
2003(69)\end{array}$ & $\begin{array}{l}11 \text { homens obesos foram submetidos a um } \\
\text { programa medicamentoso e restrição } \\
\text { calórica durante } 15 \text { semanas. Foi avaliado o } \\
\text { GEAF e sua relação com alguns hormônios. }\end{array}$ & $\begin{array}{l}\text { Redução de } 11 \% \text { no peso corporal. Ao final } \\
\text { do programa, o GEAF foi menor }(3,71 \text { kcal/ } \\
\text { min) que o predito }(4,14 \mathrm{kcal} / \mathrm{min})(\mathrm{p}<0,001) \text {. } \\
\text { Diferença associada às mudanças na } \\
\text { concentração de leptina plasmática. }\end{array}$ \\
\hline Lazzer e cols., 2004 (70) & $\begin{array}{l}26 \text { adolescentes obesos submetidos à perda } \\
\text { de peso por } 9 \text { meses, com moderada } \\
\text { restrição calórica e exercício. }\end{array}$ & $\begin{array}{l}\text { Perda de PC, MM e MG. Redução no GER, que } \\
\text { persistiu quando ajustado pela MM (6 a 12\%); } \\
\text { redução no GEAF (caminhada) de } 22 \% \text {. }\end{array}$ \\
\hline Brehm e cols., 2005 (71) & $\begin{array}{l}50 \text { mulheres obesas tratadas por } 4 \text { meses com } \\
\text { dietas pobres em CHO e LP. Foi avaliado: GER, } \\
\text { GEAF, ETA em resposta a estas dietas. }\end{array}$ & $\begin{array}{l}\text { Perda de peso nos dois grupos. Diminuição no } \\
\text { GER com perda de peso, porém sem } \\
\text { diferenças } 2 \text { e } 4 \text { meses após o final do } \\
\text { programa. }\end{array}$ \\
\hline
\end{tabular}

$\mathrm{RC}$ = restrição calórica; $\mathrm{PC}$ = peso corporal; $\mathrm{MM}=$ massa magra; $\mathrm{IMC}$ = índice de massa corpórea; $\mathrm{MG}$ = massa de gordura; $\mathrm{DEXA}=$ dual-energy $X$-ray absorptiometry; $\mathrm{GE}$ = gasto energético; $\mathrm{GET}$ = gasto energético total; $\mathrm{GER}$ = gasto energético de repouso; $\mathrm{GEB}$ = gasto energético basal; $\mathrm{GEAF}=$ gasto energético da atividade física; $E T A$ = efeito térmico da alimentação; $Q R=$ quociente respiratório. 
com a resistência à insulina (3). Sabe-se ainda que o tecido adiposo secreta moléculas importantes na regulação metabólica, como adiponectina, resistina e leptina (74). Em relação a esta última, ela é transportada na circulação e liga-se a receptores hipotalâmicos que sinalizam, em última instância, a ingestão de alimentos e a atividade simpática. Assim, o aumento da adiposidade e, portanto, maior secreção de leptina, direcionaria o organismo a aumentar seu gasto de energia e diminuir os mecanismos relacionados à fome, controlando assim os peso corporal (75). Entretanto, o que se pode constatar em humanos, diferentemente dos modelos animais, é que, na obesidade, as concentrações plasmáticas de leptina geralmente apresentam-se elevadas na mesma proporção do tecido adiposo, e que os mecanismos esperados de regulação do GE não ocorrem. Alguns autores, embora não haja um consenso definitivo, apresentam hipóteses de que a obesidade pode ser decorrente de uma resistência central à leptina (76-78).

Um dos possíveis alvos para regulação do GE pela leptina, principalmente em animais, são as proteínas desacopladoras (UCPs), possivelmente estimuladas por receptores nucleares (PPARs) no tecido adiposo marrom $(79,80)$. Essas proteínas são capazes de desacoplar o transporte de prótons na cadeia respiratória, permitindo que a energia seja perdida para o meio extramitocondrial na forma de calor. Vários tecidos humanos expressam diferentes isoformas dessa proteína, a UCP2 e a UCP3. Essas proteínas poderiam exercer a função termogênica similar à UCPl, presente no tecido adiposo marrom de animais, mas essa hipótese ainda não está totalmente esclarecida $(45,46)$. Tentando relacionar UCPs e obesidade em humanos, Yanovski e cols. (81) compararam crianças obesas e magras de várias etnias, $\mathrm{e}$ apontaram uma correlação negativa e significativa entre obesidade e expressão da UCP2.

\section{Como se comportam os componentes do GE na obesidade?}

Considerando a existência da termogênese adaptativa em obesos, sua relação com a obesidade poderia ocorrer por: a) mudanças no GER; b) mudanças no ETA; ou ainda c) mudanças no GEAF. Alguns estudos a respeito são relacionados a seguir.

\section{GER}

Pequenas mudanças no GER podem resultar desequilíbrio do BE e mudança no peso corporal em longo prazo
(82). Alguns autores, como Ravussin e cols. (83) obtiveram uma correlação significativa e negativa entre GER e peso corporal estudando os índios Pima (população indígena com alta prevalência de obesidade), por quatro anos, mostrando que um baixo GER pode ser um fator de risco para ganho de peso. Por outro lado, uma parcela considerável dos estudos mostra que quanto maior o peso corporal maior o GER, alguns destes estudos estão descritos a seguir. Luke e cols. (84) avaliando indivíduos não-obesos, observaram associação positiva entre o ganho de peso e o GER.

O maior GER em obesos parece correlacionar-se tanto com a massa magra quanto com a adiposidade (85-88). Um estudo com mulheres obesas mórbidas, divididas em três faixas de IMC (médias de 40, 49 e 60 $\mathrm{kg} / \mathrm{m}^{2}$, respectivamente), mostrou que o GED, medido por água duplamente marcada, é maior no grupo de maior IMC (89). Dionne e cols. (90) mostraram em seu estudo que o GE, particularmente a taxa metabólica basal, está significativa e positivamente relacionada com a gordura e essa correlação é ainda mais forte com o tecido adiposo abdominal. A Tabela 2 apresenta outros estudos que relacionam GER e obesidade.

\section{ETA}

O ETA também pode ser relacionado à possível termogênese adaptativa. Stock (93), há tempos, afirma que a capacidade individual a reagir com produção de calor à ingestão de alimentos define a maior ou menor propensão ao ganho de peso corporal. $\mathrm{O}$ autor define que, a partir da produção de calor os indivíduos seriam fast-burners (rápidos produtores de calor) ou slow-burners (produtores lentos de calor). Estudos mais antigos mostram que a mudança no GE após alimentação, e a combinação entre alimentação e exercício físico, é significativamente menor em indivíduos obesos do que magros (94). Maffeis e cols. (95), em um estudo com crianças obesas e magras, encontraram o ETA significativamente menor nas crianças obesas que nas crianças magras.

É importante destacar que o tipo de nutriente predominante nas refeições pode ser determinante do ETA. A menor termogênese induzida pela gordura certamente contribui para o ganho de peso, e esse fato pode ser mais pronunciado em indivíduos obesos. De acordo com Maffeis e cols. (96), dietas ricas em gorduras produziram menor ETA em um grupo de adolescentes obesas comparando a um grupo de não-obesas. Marrades e cols. (97) compararam homens jovens obe- 
Tabela 2. Relação dos estudos com GER e obesidade

\begin{tabular}{|c|c|c|}
\hline Autor & Métodos & Resultados \\
\hline $\begin{array}{l}\text { Ravussin e cols., } \\
1982(85)\end{array}$ & $\begin{array}{l}30 \text { indivíduos de } 20 \text { a } 46 \text { anos divididos em } \\
\text { trrês grupos: obesos, moderadamente } \\
\text { obesos e grupo controle. Avaliados: GER, GET } \\
\text { e composição corporal. }\end{array}$ & $\begin{array}{l}\text { Obesos tiveram GER significativamente maior } \\
\text { que os controles }(p=0,01) \text {; quando relativo } \\
\text { pela MM ou superfície corporal, não houve } \\
\text { diferenças; } Q R \text { maior nos obesos que nos } \\
\text { controles }(p<0,05) \text {; } G E T \text { maior nos grupos mais } \\
\text { obesos em valores absolutos. }\end{array}$ \\
\hline
\end{tabular}

Lazzer e cols., 2003 (91)

Foi avaliado o GET e o GER em 27

adolescentes obesos e 50 não-obesos.
A MM responde por 77,2\% do GER; todas as medidas de GE (diferentes situações) foram maiores para os obesos $(p=0,0001)$; se relativos a MM não há diferença entre obesos e magros.

GET e GER maior no grupo de maior IMC; diferenças no GE entre os grupos se correlacionam com a massa magra. Sem diferenças no ETA e no GEAF.

30 mulheres obesas com IMC médio de 48,9 $\pm 1,7 \mathrm{~kg} / \mathrm{m}^{2}$, divididas em três grupos por IMC Foi avaliado: GET, GER e ETA. Foi calculado posteriormente o GEAF.

1.088 indivíduos obesos, com IMC $\geq 35 \mathrm{~kg} / \mathrm{m}^{2}$, sendo 42 com diagnóstico de diabetes melito.

87 indivíduos obesos foram avaliados nos seguintes parâmetros: GER, composição corporal, ingestão energética. Foram divididos em três grupos, de acordo com o IMC.
GER maior nos diabéticos para homens e mulheres, mesmo quando ajustados pelas variáveis de composição corporal.

O GER não se relacionou com o IMC e sim com a massa magra.

Luis e cols., 2005 (92)

$\mathrm{RC}$ = restrição calórica; $\mathrm{PC}$ = peso corporal; $\mathrm{MM}=$ massa magra; $\mathrm{IMC}$ = índice de massa corpórea; $\mathrm{MG}=$ massa de gordura; $\mathrm{DEXA}=$ dual-energy $X$-ray absorptiometry; $\mathrm{GE}$ = gasto energético; $\mathrm{GET}$ = gasto energético total; $\mathrm{GER}$ = gasto energético de repouso; $\mathrm{GEB}$ = gasto energético basal; $G E A F=$ gasto energético da atividade física; ETA = efeito térmico da alimentação; $Q R$ = quociente respiratório.

sos e não-obesos submetidos a uma ingestão de gordura acima de $40 \%$ do valor calórico da dieta, e seus resultados mostraram que o ETA foi significativamente menor nos indivíduos obesos que nos magros. Shepard e cols. (98) submeteram indivíduos a dietas isoenergéticas, porém com diferentes proporções entre carboidratos, lipídeos ou proteínas. A ingestão aumentada de lipídeos, a despeito do mesmo valor energético da dieta, foi responsável por um menor ETA.

Sob outra perspectiva, uma dieta rica em proteínas parece estar relacionada a maior ETA (99). Johnston e cols. (100) compararam diferentes percentuais de macronutrientes da dieta e comprovaram a maior capacidade termogênica de uma dieta rica em proteínas (30\% do valor calórico total da dieta provenientes de carboidratos complexos, $10 \%$ de açúcares simples, 30\% de proteínas e $30 \%$ de gorduras), quando comparada com uma dieta rica em carboidratos ( $50 \%$ do valor calórico total da dieta provenientes de carboidratos complexos, 10\% de açúcares simples, 15\% de proteínas e $25 \%$ de gorduras).

A própria distribuição irregular do número e do va lor energético das refeições pode interferir no ETA. Farshchi e cols. (101) avaliaram nove indivíduos com peso normal em um estudo de duas etapas: na primeira foi realizado número regular de refeições, em horários preestabelecidos. Na outra etapa, os indivíduos mudaram o número de refeições de maneira aleatória (dieta irregular), porém mantendo o mesmo valor energético total ingerido. O ETA foi significativamente menor após período irregular, o que pode significar elevação do peso corporal no longo prazo. 
Os diferentes estudos, analisando o ETA, podem apresentar até $48 \%$ de variabilidade individual, porquanto o nível de atividade física, a glicemia e a resistência insulínica explicam $15 \%$ dessa variabilidade. A hiperglicemia e a hiperinsulinemia promovem redução na atividade simpática, o que pode explicar a redução na termogênese (102-105).

\section{GEAF}

A atividade física parece colaborar com o aumento, ou pelo menos a preservação da massa magra, proporcionando elevação ou ao menos a manutenção do GE (106-108). Entretanto, nem todos os estudos são concordantes nesse sentido (17,109-111). Hipotetiza-se a possibilidade de o GEAF estar diminuído em indivíduos obesos. Froidevaux e cols. (112) mostram que há diminuição no GEAF em resposta à perda de peso, e que essa diminuição está atribuída à diminuição no trabalho executado após a perda de peso corporal. Adolescentes obesos apresentaram menor GEAF e GED, quando comparados a adolescentes magros, em valores absolutos e relativos à massa magra (91). Em outro estudo com meninos adolescentes submetidos a um programa de perda de peso, o GEAF (caminhada) no final do programa, mesmo quando ajustado pelo peso corporal e massa magra, foi significativamente menor do que no início do programa (70). No mesmo raciocínio, Doucet e cols. (69) observaram uma diminuição no GEAF após um programa para perda de peso em homens.

Segal e cols. (113) compararam homens de IMC similares, porém com composição corporal diferentes. Os autores observaram que o GER e o ETA, durante e após o exercício, eram maiores para os indivíduos com menor percentual de gordura $(<15 \%)$ do que nos com maior adiposidade $(>25 \%)$, indicando que a composição corporal é fator determinante da termogênese. Weinsier e cols. (58) não encontraram diferenças no GEAF após a perda de peso em mulheres brancas e negras. Porém, estes mesmos autores, dois anos mais tarde (114), estudaram mulheres que mantiveram ou não o peso corporal após o programa para perda de peso. Os resultados apontaram que as mulheres que mantiveram o peso apresentavam um GEAF $42 \%$ maior do que as mulheres que recuperaram peso (mais que $6 \mathrm{~kg} / \mathrm{ano}$ ).

Rosembaum e cols. (115) procuraram identificar adaptações do GE após variação de peso corporal, hipotetizando que a adaptação do GE poderia ocorrer não no exercício físico em si, mas nas atividades de baixa intensidade, ou seja, nas atividades cotidianas. Para essa investigação, os autores avaliaram a eficiência do trabalho muscular (a partir de testes em cicloergômetro e ressonância magnética nuclear no músculo). O aumento de peso corporal foi responsável por um GE 35\% menor para realização das atividades de baixa intensidade.

\section{CONSIDERAÇÕES A RESPEITO DOS ESTUDOS APRESENTADOS}

Como se pode observar, para os três componentes do GED, os resultados ainda são bastante controversos. Muitas vezes, nota-se que diferentes resultados entre os estudos decorrem de diferentes técnicas de análise empregadas, ou ainda do controle dos procedimentos experimentais. Vários autores $(17,61,116-119)$ procuram enumerar os possíveis fatores que colaboram com essa controvérsia, entre os quais pode-se destacar:

A. A técnica empregada (água duplamente marcada, calorimetria de sala, circuitos abertos e fechados). Os métodos realizados em laboratório (calorimetria indireta ou calorímetros de sala) podem subestimar as medidas em até $20 \%$ em indivíduos sedentários, e em até $68 \%$ em atletas treinados.

B. Os procedimentos utilizados na técnica de calorimetria: medidas feitas de modo contínuo ou intermitente, em diferentes períodos; troca gasosa analisada em uma tomada única ou repetições de medida (o coeficiente de variabilidade pode chegar até a $20 \%$ ); equações para transformar troca gasosa em quilocalorias (podem implicar erro de até 3\%); controle de linhas de base apropriada, principalmente quando se avaliam respostas termogênicas à dieta. Nas medidas do ETA, de modo geral, observa-se dois momentos bem diferenciados: o aumento pós-prandial do GE, que implicaria ingestão, digestão e absorção dos nutrientes, e, ainda, o GE em momentos posteriores, para transporte, captação e metabolismo específico desses nutrientes (120).

C. A padronização das refeições para se determinar o ETA. Mesmo tendo sido estabelecido o menor efeito termogênico dos lipídeos, a proporção entre os diferentes tipos de gordura pode gerar controvérsia. Alguns autores verificaram elevação do ETA após a ingestão de ácidos graxos monoinsaturados (121), do ácido graxo eicosapentanóico (122), entre outros. Além dis- 
so, o valor energético da refeição administrada, o tempo para estabilização do peso corporal, e a composição da refeição administrada variam muito entre os diferentes estudos (120).

D. As técnicas de composição corporal utilizadas para definir os sujeitos como obesos ou não-obesos, ou ainda para expressar os resultados em relação à massa magra. Ravussin e cols. (85) encontraram GER significativamente maior em indivíduos obesos em valores absolutos, o que significa que quanto maior a quantidade de massa magra, maior o GER. Quando os valores foram expressos por quilograma de massa magra, não foram encontradas diferenças entre obesos e não-obesos.

E. A relação do GE com resistência à insulina, nível de atividade física praticado, idade ou dieta realizada anteriormente aos testes.

\section{CONCLUSÃo}

Várias são as técnicas existentes para avaliação do GE, e a escolha do melhor método dependerá dos objetivos da pesquisa.

No que diz respeito à comparação do GE entre indivíduos não-obesos e obesos, os resultados apontam para um comportamento diferenciado desses últimos, provavelmente, em virtude dos constantes processos de restrição energética a que esses indivíduos estão sujeitos. Entretanto, não se conseguiu ainda identificar em qual componente específico do GE esse comportamento diferenciado pode ocorrer (no GER, no ETA ou no GEAF) e por quais mecanismos, sejam eles hormonais, neuronais ou moleculares.

\section{AGRADECIMENTOS}

Os autores expressam seu agradecimento à Fundação de Amparo à Pesquisa do Estado de São Paulo (Fapesp), pelo financiamento do projeto de pesquisa (Processo no 03/08689-0) e ao Conselho Nacional de Desenvolvimento Científico e Tecnológico (CNPQ), pela bolsa concedida.

\section{REFERÊNCIAS}

6. Horvath TL. The hardship of obesity: a soft-wired hypothalamus. Nat Neurosci. 2005;8(5):561-5.

7. World Health Organization. Diet, nutrition and the prevention of chronic disease. WHO Technical Report Series 916, 2003.
8. Roth J, Qiang X, Marbán SL, Redelt H, Lowell BC. The obesity pandemic: where have we been and where are we going? Obes Res. 2004;12(Suppl): 88S-101.

9. Orzano AJ, Scott JG. Diagnosis and treatment of obesity in adults: an applied evidence-based review. J Am Board Fam Pract 2004; 17(5): 359-69.

10. IBGE - Instituto Brasileiro de Geografia e Estatística. Pesquisa de orçamentos familiares 2002-2003. Ministério da Saúde, 2004.

11. Astrup A, Gotzsche PC, Werken K, Ranneires C, Toubro S, Raben A, Buemann B. Meta-analysis of resting metabolic rate in formerly obese subject. Am J Clin Nutr. 1999;69(6):1117-22.

12. Heymsfield SB, Darby PC, Muhlheim LS, Gallagher D, Wolper C, Allison DB. The calorie: myth, measument an reality. Am J Clin Nutr. 1995;62(Suppl):1034S-41.

13. Hill JO. Understanding and addressing the epidemic of obesity: an energy balance perspective. Endocr Rev. 2006;27(7):750-61.

14. Erik D, Salazar G, Saavedra C, Tirapegui J. Cap 23: gasto energético e atividade física. In: Tirapegui J, organizador. Nutrição, metabolismo e suplementação na atividade física. São Paulo: Atheneu; 2006.

15. Pedrosa RG, Donato Jr J, Araújo Jr JA, Tirapegui J. Gasto energético: componentes, fatores determinantes e mensuração. In: Angelis RC, Tirapegui J, organizador. Fisiologia da nutrição humana: aspectos básicos, aplicados e funcionais. São Paulo: Atheneu; 2007.

16. Diener JRC. Calorimetria indireta. Rev Ass Med Brasil. 1997;43(3): 245-53.

17. Nahas MV. Introdução: por que medir atividades físicas habituais? In: Barros MVB, Nahas MV, organizadores. Medidas da atividade física: teoria e aplicação em diversos grupos populacionais. Londrina: Midiograf; 2003. p. 9-15.

18. Pi-Sunyer FX. Overnutrition and undernutrition as modifiers of metabolic process in disease states. Am J Clin Nutr. 2000, 72(Suppl):533S-7.

19. Wahrlich V, Anjos LA. Aspectos históricos e metodológicos da medição e estimativa da taxa metabólica basal: uma revisão da literatura. Cad. Saúde Pública. 2001;17(4):801-17.

20. Lee RD, Nieman DC. Nutritional assessment. $2^{2}$ ed. St Louis: Mosby; 1995.

21. Suen V, Silva, GA, Marchini JS. Determinação do metabolismo energético no homem. Medicina Ribeirão Preto. 1998;31:13-21.

22. Toth MJ, Poehlman ET. Effects of exercise on daily energy expenditure. Nutr Rev. 1996;54(4-II):S140-8.

23. Jequier $E$, Schutz $Y$. Long-term measurements of energy expenditure in humans using a respiratory chamber. Am J Clin Nutr. 1983;38(6):989-98.

24. McArdle WD, Katch FI, Katch VL. Fisiologia do exercício: energia, nutrição e desempenho humano. $4^{\mathrm{a}}$ ed. Rio de Janeiro: Guanabara Koogan; 1998.

25. Severi S, Malavolti M, Battistini N, Bedogni G. Some applications of indirect calorimetry to sports medicine. Acta Diabetol. 2001;38(1):23-6.

26. Yamamura C, Tanaka S, Futami J, Oka J, Ishikawa-Takata $\mathrm{K}$ Kashiwazaki H. Activity diary method for predicting energy expenditure as evaluated by a whole-body indirect human calorimeter. J Nutr Sci Vitaminol. 2003;49(4):262-9.

27. Scagliusi FB, Lancha Jr. AH. Estudo do gasto energético po meio da água duplamente marcada: fundamentos, utilização e aplicações. Rev Nutr. 2005;18(4):541-51.

28. Craig $C L$, Marshall $A L$, Sjostrom $M$, Bauman $A E$, Booth $M L$, Ainsworth BE, et al. International physical activity question- 
naire: 12-country reability and vality. Med Sci Sports Exerc. 2003;35(8):1381-95.

29. Matsudo S, Aratujo T, Matsudo V, Andrade D, Andrade E, Oliveira $\mathrm{LC}$, et al. Quastionário internacional de atividade física (IPAQ): estudo de validade e reprodutibilidade no Brasil. Rev Bras Ativ Fís Saúde. 2001;6(2):5-18.

30. Bouchard C, Tremblay A, Leblanc C, Lortie G, Savard R, Thériault G. A method to assess energy expenditure in children and adults. Am J Clin Nutr. 1983;37(3)461-7.

31. OMS - Organização Mundial da Saúde. Necessidades de energia e proteína. Série de relatos técnicos 724. São Paulo: Roca, 1998. 225p.

32. Mattes RD, Bormann LA. Reduced dietary underrecording with concurrent traccking of hunger. J Am Diet Assoc. 2001;101(5):578-80.

33. Goris AHC, Westerterp-Plantenga MS, Westerterp KR. Undereating and underrecording of habitual food intake in obese men:selectie underreporting of fat intake. Am J Clin Nutr. 2000;71(1):130-4.

34. Harris J, Benedict FG. A biometric study of basal metabolism in man. Boston: Carnegie Institute of Washington; 1919.

35. FAO (Food and Agriculture Organization)/WHO (World Healft Organization)/UNU (United Nations University). Energy and protein requirements. WHO Techinical Report Series $724, \mathrm{Ge}-$ neva: WHO, 1985.

36. Dobratz RJ, Sibley SD, Beckman TR, Valentine BJ, Kellogg TA Ikramuddin S, et al. Predicting energy expenditure in extremely obese women. J Parenter Enteral Nutr. 2007;31:217-27.

37. US National Academy of Sciences (USA). Dietary reference intakes: applications in dietary assessment. Washington, DC: National Academy press; 2000.

38. Oliveira ESA, Pires EAG, De Bem MFL. Medidas de atividades físicas em adolescentes. In: Barros MVB, Nahas MV, organizadores. Medidas da atividade física: teoria e aplicação em diversos grupos populacionais. Londrina: Midiograf; 2003. p. 71-9.

39. Ekelund ULF, Yngve A, Brage S, Westerterp K, Sjostron M. Body movement and physical activity energy expenditure in children and adolescents: how to adjust for differences in body size and age. Am J Clin Nutr. 2004;79(5):851-6.

40. Thompson DL, Rakow J, Perdue SM. Relationship between accumulated walking and body composition in middle-aged woman. Med Sci Sports Exerc. 2004;36(5):911-4.

41. Schultz Y, Weinsier R, Hunter GR. Assessment of free-living physical activity in humans: an overview of currently available and proposed new measures. Obes Res. 2001;9(6):368-79.

42. Lifton N, Gordon GB, Visscher MB, Nier AO. The fate of utilized molecular oxygen and the source of the oxygen of respiratory carbon dioxide, studied with the aid of heavy oxygen. J Biol Chem. 1949;180:803-11.

43. Speakman JR. The history and theory of the doubly labeled water technique. Am J Clin Nutr. 1998;68(Suppl): 932S-8.

44. Ainslie P, Reilly T, Westerterp K. Estimating human energy expenditure: a review of techniques with particular reference to doubly labelled water. Sports Med. 2003;33(9):683-98.

45. Levine JA. Measurement of energy expenditure. Public Health Nutr. 2005;8(7A):1123-32.

46. Schoeller DA. The importance of clinical research: the role of thermogenesis in human obesity. Am J Clin Nutr. 2001; 73(3):511-6.

47. Astrand P. Tratado de fisiologia do exercício. $2^{\mathrm{a}}$ ed. Rio de Janeiro: Interamericana; 1977.
48. Zhang Y, Proença R, Maffei M, Baroni M, Leopold L, Friedman JM. Positional cloning of the mouse obese gene and its human homologue. Nature. 1994;372(6505):425-32.

49. Lafontan M. Fat cells: afferent and efferent messages define new approaches to treat obesity. Annu Rev Pharmacol Toxicol. 2005;45:119-46.

50. Dulloo AG, Samec S. Uncoupling proteins: do they have a role in body weight regulation? News Physiol Sci. 2000;15:313-8.

51. Hesselink MKC, Mensink M, Schrawen P. Human uncoupling protein-3 and obesity: an update. Obes Res. 2003;11(12):1429-43.

52. Fantino $\mathrm{M}$, Cabanac $\mathrm{M}$. Body weight reglation with a proportional hoarding response in rat. Physiol Behav. 1980;24(5):934-42.

53. Fantino $M$, Boucher $H$, Facon $F$, Mathiot P. Dexfenfluramine ansd body weight regulation: experimental study with hoarding behaviour. Clin Neuropharmacol. 1988;11(Suppl 1):S97-104.

54. MacLean PS, Higgens JH, Johnson GC, Flemming-Elder BK, Donahoo WT, Melanson E, et al. Enhanced metabolic efficiency contributes to weight regain after weight loss in obesityprone rats. Am J Physiol. 2004;287(6): R1306- 15.

55. Rising R, Lifshitz F. Energy expenditures and physical activity in rats with chronic suboptimal nutrition. Nutrition and Metabolism. 2006;31:3-11.

56. Wyatt HR, Grunwald GK, Seagle HM, Klem ML, McGuire MT, Wing RR, et al. Resting energy expenditure in reduced-obese subjects in the National Weight Control Registry. Am J Clin Nutr. 1999;69(6):1189-93.

57. Major GC, Doucet E, Trayhurn P, Astrup A, Tremblay A. Clinical significance of adaptive thermogenesis. Intern J Obes. 2007;31(2):204-12.

58. Keen $\mathrm{H}$, Thomas BJ, Jarret RJ, Fuller JH. Nutrient intake, adiposity, and diabetes. Britsh J Med. 1979;10(1):655-8.

59. Braitman LE, Adlin EV, Stanton JL. Obesity and caloric intake: the National Health and Nutrition Examination Survey of 1971-1975 (HANES I). J Chronic Dis. 1985;38(9):727-32.

60. Shah M, Miller DS, Geissler CA. Lower metabolic rates of post-obese versus lean women: thermogeneses, basal metabolic rate and genetics. Eur J Clin Nutr. 1988;42(9):741-52.

61. Weinsier RL, Nagy TR, Hunter GR, Darnell BE, Hensrud DD, Weiss, HL. Do adaptative changes in metabolic rate favor weight regain in weight reduced individuals? An examination of the set-point theory. Am J Clin Nutr. 2000;72(5):1088-94.

62. Weinsier RL, Hunter GR, Zuckerman PA, Darnell BE. Low resting and sleeping energy expenditure and fat use do not contribute to obesity in women. Obes Res. 2003;11(8):937-44.

63. Lof $\mathrm{M}$, Forsum $\mathrm{E}$. Validation of energy intake by dietary recall against different methods to assess energy expenditure. $J$ Hum Nutr Dietet. 2004;17:471-80.

64. Scagliusi FB, Lancha Junior AH. Subnotificação da ingestão energética na avaliação do consumo alimentar. Rev Nutr. 2003;16:471-81.

65. Maurer J, Taren DL, Teixeira PJ, Thomson CA, Lohman TG, Going SB, et al. The psychosocial and behavioral characteristics related to energy misreporting. Nutr Rev. 2006;64:53-64.

66. Leibel RL, Rosenbaum M, Hirsch J. Changes in energy expenditure resulting from altered body weight. $\mathrm{N}$ Engl J Med. 1995;332:621-8. Erratum in: N Engl J Med. 1995;333:399.

67. Doucet $E$, St-Pierre S, Almerás N, Després JP, Bouchard C, Tremblay A. Evidence for existence of adaptive thermogenesis during weight loss. Britsh J Nutr. 2001;85:715-23. 
68. Weyer C, Walford RL, Harper IT, Milner M, MacCallum T, Tataranni PA, et al. Energy metabolism after 2 y of energy restriction: the biosphere 2 experiment. Am J Clin Nutr. 2000;72(4):946-53.

69. Dulloo AG, Jacquet J, Guardier L. Poststarvation hyperphagia and body fat overshooting in humans: a role for feedback signals from lean and fat tissues. Am J Clin Nutr. 1997;65(3):717-23.

70. Weinsier RL, Hunter GR, Zuckerman PA, Redden DT, Darnell $\mathrm{BE}$, Larson $\mathrm{DE}$, et al. Energy expenditure and free-living physical activity in black and white women: comparison before and after weight loss. Am J Clin Nutr. 2000;71(5):1138-46.

71. Doucet $E$, St-Pierre $S$, Alméras $N$, Mauriège $P$, Rchard $D$, Tremblay A. Changes in energy expenditure and substrate oxidation resulting from weight loss in obese men and women: Is there an important contribution of leptin? J Clin Endocrinol Metab. 2000;85(4):1550-6.

72. Kempen KP, Saris WH, Westerterp KR. Energy balance during an 8-wk energy-restricted diet with and without exercise in obese women. Am J Clin Nutr. 1995;62(4):722-9.

73. Foster GD, Wadden TA, Swain RM, Anderson DA, Vogt RA. Changes in resting energy expenditure after weight loss in obese African American and white women. Am J Clin Nutr. 1999;69(1):13-7.

74. Doucet $E$, Imbeault $P$, St-Pierre $S$, Almerás $N$, Maurège $P$, Després JP, et al. Greater than predicted decrease in energy expenditure during exercise after body weight loss in obese men. Clin Sci. 2003;105(1):89-95.

75. Lazzer S, Boirie Y, Montaurier C, Vernet J, Meyer M, Vermorel $M$. A weight reduction program preserves fat-free mass but not metabolic rate in obese adolescents. Obes Res. 2004;12(2):233-40.

76. Brehm BJ, Spang SE, Lattin BL, Seeley RJ, Daniels SR, $D^{\prime}$ Alessio DA. The role of energy expenditure in the differential weight loss in obese women on low-fat and low-carbohydrate diets. J Clin Endocrinol Metab. 2005;90(3):1475-82.

77. Dulloo AG. Editorial: suppressed thermogenesis as a cause for resistance to slimming and obesity rebound: adaptation or illusion? Int J Obes. 2007;31(2):201-3.

78. Dulloo AG, Jacquet J. An adipose-specific control of thermogenesis in body weight regulation. Int $\mathrm{J}$ Obes Relat Metab Disord. 2001;25(Suppl 5):S22-9.

79. Alaniz MHF, Takada J, Vale MICA, Lima FB. O tecido adiposo como órgão regulador do metabolismo. Arq Bras Endocrinol Metab. 2006;50(2):216-29.

80. Bouret SG, Simerly RB. Minireview: leptin and development of hypothalamic feeding circuits. Endocrinology. 2004;145(6): 2621-6.

81. Munzberg $H$, Myers MG. Molecular and anatomical determinants of central leptin resistance. Nat Neurosc. 2005;8(5):566-70.

82. Chan JL, Heist K, DePaoli AM, Veldhuis JD, Mantzoros CS. The role of falling leptin levels in the neuroendocrine and metabolic adaptation to short-term starvation in healthy men. J Clin Invest. 2003;111:1409-21.

83. Ribeiro SML, Santos, ZA, Silva, RJ, Louzada E, Donato Jr J, Tirapegui J. Leptina: aspectos sobre o balanço energético, exercício físico e amenorréia do esforço. Arq Bras Endocrinol Metab. 2007;51(1):11-24.

84. Kelly LJ, Vicario PP, Thompson GM, Candelore MR, Doebber TW, Ventre J, et al. Perixome proliferators-activated receptor $\gamma$ and $\beta$ mediate in vivo regulation of uncoupling protein (UCP1, UCP2, UCP3) gene expression. Endocrinology. 1998; 139(12):4920-7.
85. Yang X, Downes M, Yu RT, Bookout AL, He W, Straume M, et al. Nuclear receptor expression links the circadian clack to metabolism. Cell. 2006;126(4):801-10.

86. Yanovski JA, Diament AL, Sovik KN, Nguyen TT, Li H, Sebring NG, et al. Associations between uncoupling protein 2, body composition and resting energy expenditure in lean and obese African American, white and Asian. Am J Clin Nutr. 2000;71(6):1405-12.

87. Huang K, Kornas N, Steinbeck K, Loughnan G, Caterson I D. Resting metabolic rate in severely obese diabetic an nondiabedic subjects. Obes Res. 2004;12(5):840-45.

88. Ravussin E, Lillioja S, Knowler WC, Christin L, Freymond D, Abbott WG, et al. Reduced rate of energy expenditure as a risk factor for body-weight gain. $\mathrm{N}$ Engl $\mathrm{J}$ Med. 1988;318(8):467-72.

89. Luke A, Durazo-Avisu R, Cao G, Adeyesso A, Tayo B, Cooper R. Positive association between resting energy expenditure and weight gain in a lean adult population. Am J Clin Nutr. 2006;83(5):1076-81.

90. Ravussin E, Burnand B, Schutz Y, Jéquier E. Twenty-four-hour energy expenditure and resting metabolic rate in obese, moderately obese, and control subjects. Am J Clin Nutr. 1982;35(3):566-73.

91. Jéquier E, Tappy L. Regulation of body weight in humans. Physiol Rev. 1999;79(2):451-80.

92. Prentice AM, Black AE, Coward WA, Cole TJ. Energy expenditure in overweight and obese adults in affluent societies: an analysis of 319 doubly-labelled water measurements. Eur $\mathrm{J}$ Clin Nutr. 1996;50(2):93-7.

93. Schulz LO, Schoeller DA. A compilation of total daily energy expenditures and body weights in healthy adults. Am J Clin Nutr. 1994;60:676-81.

94. Das SK, Saltzman E, McCrory MA, Hsu LKG, Shikora SA, Dolnikowski $\mathrm{G}$, et al. Energy expenditure is very high in extremely obese women. J Nutr. 2004;134(6):1412-6.

95. Dionne I, Van Vugt S, Tremblay A. Postexercise macronutrient oxidation: a factor dependent on postexercise macronutrient intake. Am J Clin Nutr. 1999;69(5):927-30.

96. Lazzer S, Boirie Y, Bitar A, Montaurier C, Vernet J, Meyer M, et al. Assessment of energy expenditure associated with physical activities in free-living obese and nonobese adolescents. Am Clin Nutr. 2003;78(3):471-9.

97. Luis DA, Izaola RAO, Sagrado MG, Conde R. Resting energy expenditure, cardiovascular risk factors and insulin resistance in obese patients. An Nutr Metab. 2005;49(6):381-5.

98. Stock MJ. Gluttony and thermogenesis revisited. Int J Obes. 1999;23(11):5-17.

99. Segal KR, Gutin B. Thermic effects of food and exercise in lean and obese woman. Metabol Clin. 1983;32(6):581-9.

100. Maffeis C, Schutz Y, Zoccante L, Micciolo R, Pinelli L. Mealinduced thermogenesis in lean and obese prepubertal children. Am J Clin Nutr. 1993;57(4):481-5.

101. Maffeis $C$, Schutz Y, Grezzani A, Provera S, Piacentini G, Tatò L. Meal-induced thermogenesis and obesity: is a fat meal a risk factor for fat gain in chilren? J Clin Endocrinol Metabol. 2001;86(1):214-9.

102. Marrades MP, Martinéz JA, Moreno-Aliaga MJ. Differences in short-termo metabolic responses to a lipid load in lean (resistant) vs obese (suceptible) young male subjects with habitual high-fat consumption. Eur J Clin Nutr. 2007;61(2):166-74.

103. Shepard TY, Weil KM, Sharp TA. Occasional physical inactivity combined with a high fat diet may be important in the 
development and maintenance of obesity in human subjects. Am J Clin Nutr. 2001;73(4):703-8.

104. Luscombe-Marsh ND, Noakes M, Wittert GA, Keogh JB, Foster P, Clifton PM. Carbohydrate-restricted diets in either monounsaturaded fat or protein are equally effective at promoting fat loss and improving blood lipids. Am J Clin Nutr. 2005;81(4):762-72.

105. Johnston CS, Day CS, Swan PD. Postprandial thermogenesis is increased $100 \%$ on a high-protein, low-fat diet versus a high-carbohydrate, low-fat diet in healthy, young women. J Am Coll Nutr. 2002;21(1):55-61.

106. Farshchi HR, Taylor MA, Macdonald IA. Decreased thermic effect of food after an irregular compared with a regular meal pattern in healthy lean women. Int J Obes. 2004;28(5):653-60.

107. De Jonge L, Bray G. The thermic effect of food and obesity: a critical review. Obes Res. 1997;5(6):622-31.

108. Tataranni PA, Larson DE, Snitker S, Ravussin E. Thermic effects of food in humans: methods and results from use of a respiratory chamber. Am J Clin Nutr. 1995;61(5):1013-9.

109. Ravussin E, Bogardus C, Schwartz R. Thermic effect of infused glucose and insulin in man: decreased response with increased insulin resistance in obesity and noninsulin-dependent diabetes mellitus. J Clin Invest. 1983;72(3):893-902.

110. Den Besten C, Vansant G, Westrate J, Deurenberg P. Resting metabolic rate and diet-induced thermogenesis in abdominal and gluteal-femoral obese women before and after weight reduciotn. Am J Clin Nutr. 1988;47(5):804-7.

111. Stiegler $P$, Cunliffe $A$. The role of diet and exercise for the maintenance of fat free mass and resting metabolic rate during weight loss. Sports Med. 2006;36(3):239-62.

112. Bingham SA, Goldberg GR, Coward DA, et al. The effect of exercise and improved physical fitness on basal metabolic rate. $\mathrm{Br} \mathrm{J}$ Nutr. 1989;61(2):155-73.

113. Meijer GA, Janssen GME, Westerterp KR, et al. The effect of a 5-month endurance training programme on physical activity: evidence for a sex-difference in the metabolic response to exercise. Eur J Appl Physiol. 1991;62(1):11-7.

114. Buemann B, Astrup A, Christensen NJ. Three months aerobic training fails to affect 24-hour energy expenditure in weight stable, post-obese women. Int J Obes. 1992;16(10):809-16.

115. Poehlman ET, Gardner AW, Ades PA, Katzman-Rooks SM, Montgomery SM, Atlas OK, et al. Resting energy metabolism and cardiovascular disease risk in resistance-trained and aerobically trained males. Metabolism. 1992;41(12):1351-60.

116. Ballor DL, Pehlman ET. Resting metabolic rate and coronaryheart-disease risk factors in aerobically and resistance-trained women. Am J Clin Nutr. 1992;56(6):968-74.
117. Froidevaux F, Schutz Y, Christin L, Jéquier E. Energy expenditure in obese women before and during weight loss, after refereeing, and in the weight-relapse period. Am J Clin Nutr. 1993;57(1):35-42.

118. Segal KR, Gutin B, Nyman AM, Pi-Sunyer X. Thermic effect of food at rest, during exercise, and after exercise in lean and obese men of similar body weight. J Clin Invest. 1985;76(3):1107-12.

119. Weinsier RL, Hunter GR, Desmond RA, Byrne NM, Zuckerman PA, Darnell BE. Free-living activity energy expenditure in women successful and unsucessful at mantaining a normal body weight. Am J Clin Nutr. 2002;75(3):499-504.

120. Rosembaum M, Vanderborne K, Goldsmith R, Simoneau JA, Heymsfield S, Joanisse DR, et al. Effects of experimental weight perturbation on skeletal muscle work efficiency in human subjects. Am J Physiol Regul Integr Comp Physiol. 2003; 285(1):R183-92.

121. Houde-Nadeau M, DeJonge L, Garrel D. Thermogenic response to food: intra-individual variability and measurement reliability. J Am Coll Nutr. 1993;12(5):511-6.

122. Schulz LO, Alger S, Harper I, et al. Energy expenditure of elite female runners measured by respiratory chamber and doubly lasbeled water. J Appl Physiol. 1992;72(1):28-38.

123. Piers LS, Soares M, Makan T, Shetty P. Thermic effect of a meal: 1. methodology and variation in normal young adults. Br J Nutr. 1992;67(2):165-75.

124. Soares MJ, Sheela M, Kurpad A, et al. The influence of different methods on basal metabolic rate measurements in human subjects. Am J Clin Nutr. 1989;50(4):731-6.

125. Granata GP, Brandon LJ. The thermic effect of food and obesity: discrepant results and methodological variations. Nutr Rev. 2002;60(8): 223-33.

126. Piers LS, Walker KZ, Stoney RM, Soares MJ, O'Dea K. The influence of type of dietary fat on postprandial fat oxidation rates: monounsaturated (olive oil) vs saturated fat (cream). Int J Obes Relat Metab Disord. 2002; 26:814-21.

127. Armstrong MB, Towle HC. Polyunsaturated fatty acids stimulate hepatic UCP2 expression via a PPARalpha-mediated pathway. Am J Physiol Endocrinol Metab. 2001;281(6):E1197-204.

Endereço para correspondência:

Sandra Maria Lima Ribeiro

Escola de Artes, Ciências e Humanidades da USP

Av. Arlindo Bettio, 1000

03828-000, São Paulo SP

E-mail: smlribeiro@uol.com.br 